\title{
Properties of the effective Hamiltonian for the system of neutral kaons*
}

\author{
Justyna Jankiewicz \\ Institute of Physics, University of Zielona Góra, Prof. Z. Szafrana 4a, Zielona Góra, Poland \\ e-mail: J.Jankiewicz@proton.if.uz.zgora.pl
}

(Rec. 20 June 2005)

\begin{abstract}
We study the properties of time evolution of the $K^{0}-\bar{K}^{0}$ system in spectral formulation. Within the one-pole model we find the exact form of the diagonal matrix elements of the effective Hamiltonian for this system. It appears that, contrary to the Lee-Oehme-Yang (LOY) result, these exact diagonal matrix elements are different if the total system is CPT-invariant but CP-noninvariant.
\end{abstract}

Key words: neutral $\mathrm{K}$ mesons, the $\mathrm{CPT}$ invariance, the $\mathrm{CP}$ noninvariance, the effective Hamiltonian, the one-pole model, the LOY model

\section{INTRODUCTION}

The realistic model of the pair of unstable neutral particles (neutral $K$ mesons) is discussed. The model is based on the assumed properties of the spectral function defining the evolution operator. It is assumed that this spectral function describing the mass distribution has the Breit-Wigner form. This allows one to express the matrix elements of the evolution operator in terms of the combinations of exponential integral functions. Then, these matrix elements of the evolution operator are used to calculate the matrix elements of the effective Hamiltonian governing the evolution of a two-state quantum system corresponding to the $K-\bar{K}$ mesons and similar systems.

The aim of the paper was to find the analytic expression for the difference of the diagonal elements of the effective Hamiltonian with the use of the exponential integral functions. As a result, the relatively complex physical model has been reduced to a form which can be immediately used for computer simulations exploring the behaviour of this very important difference with the change in time $(t)$, given the numerical values of the parameters characterising the system (mass, lifetime etc.).

Furthermore, a method for extracting asymptotic values of observable parameters (like the difference mentioned above) from general, analytic expressions, in the case of two quantum objects with very different lifetimes, has been developed.

The computational methods used are of universal character and might be used outside the elementary particles. As our main interest is the research of the behaviour of the exact expressions describing the system, symbolic methods were used. Additionally, some of the parts of the system are of highly oscillatory nature, so purely numerical methods might be inappropriate here.
This paper is addressed not only to high energies physicists but also to physicists who are searching for properties of two or more levels (or particles) systems in atomic or molecular physics, etc. The problem of the CPT invariance or the CPT noninvariance applies not only in the case of neutral mesons but also, for example, in the case of electron-positron pairs [1]. For this reason Section Preliminaries (based on [2]) appears in the paper. This Section familiarizes Readers with the mentioned problem.

The computational part was carried out with the use of a symbolic package, namely Mathematica, and the functional-objective characteristics of its programming language were fully used. The symbolic approach enabled us to use both the objects and methods built into Mathematica (like the exponential integral functions, the asymptotic procedures etc.) as well as define our own objects and symbolic methods used in the calculations (like the effective meson vectors with scalar products, norms, the operation of the effective Hamiltonian etc.). Whenever we failed to come up with a suitable symbolic method, the object was wrapped and left intact.

This approach has the advantage that all calculations are carried out exactly, perhaps leaving some parts unevaluated and thus avoiding approximations. Mathematica gives its user the possibility to generate code in a low-level language ( $\mathrm{C}$, Fortran), so now, having arrived at the final formulae it will be simple to produce efficient and fast, and at the same time as exact as possible, numerical programs for investigating various neutral mesons on various time-scales and in various conditions.

The paper is organized as follows. In Sec. 2 we review briefly the Lee-Oehme-Yang and other methods of description of neutral $K$ system. Section 3 describes the spectral model of time evolution in neutral mesons subspace. In Sec. 4 the difference of the diagonal matrix elements of the

*Paper presented at The XXXVI Symposium on Mathematical Physics, Poster session, Toruń, Poland, June 9-12, 2004. 
effective Hamiltonian is calculated. The concluions are contained in Sec. 5.

\section{PRELIMINARIES}

Following the LOY approach, a nonhermitian Hamiltonian $H_{\|}$is usually used to study the properties of the particle-antiparticle unstable system [3-7]

$$
H_{\|} \equiv M-\frac{i}{2} \Gamma
$$

where

$$
M=M^{+}, \quad \Gamma=\Gamma^{+}
$$

are $(2 \times 2)$ matrices acting in a two-dimensional subspace $\mathcal{H}_{\|}$of the total state space $\mathcal{H}$. The $M$-matrix is called the mass matrix and $\Gamma$ is the decay matrix. Lee, Oehme and Yang derived their approximate effective Hamiltonian $H_{\|} \equiv H_{\text {LOY }}$ by adapting the one-dimensional Weisskopf-Wigner (WW) method to the two-dimensional case corresponding to the neutral kaon system.

Almost all properties of this system can be described by solving the Schrödinger-like equation [3-6]

$$
i \frac{\partial}{\partial t}|\psi ; t\rangle_{\|}=H_{\|}|\psi ; t\rangle_{\|}, \quad\left(t \geq t_{0}>-\infty\right)
$$

(where we have used $\hbar=c=1$ ) with the initial conditions

$$
\|\left|\psi ; t=t_{0}\right\rangle_{\|} \|=1, \quad\left|\psi ; t<t_{0}\right\rangle_{\|}=0
$$

for $\left|\varphi ; t=t_{0}\right\rangle_{\|}$belonging to the subspace of states $\mathcal{H}_{\|}$ $\left(\mathcal{H}_{\|} \subset \mathcal{H}\right)$, spanned by, e.g., orthonormal neutral kaons states $K^{0}$ and $\bar{K}^{0}$. The solutions of Eq. (3) may be written in a matrix form, which may be used to define the time evolution operator $U_{\|}(t)$ acting in subspace $\mathcal{H}_{\|}$

$$
|\psi ; t\rangle_{\|}=U_{\|}(t)\left|\psi ; t_{0}=0\right\rangle_{\|} \equiv U_{\|}(t)|\psi\rangle_{\|},
$$

where

$$
|\psi\rangle_{\|} \equiv a_{1}|\mathbf{1}\rangle+a_{2}|\mathbf{2}\rangle
$$

and $|\mathbf{1}\rangle$ denotes particle "1" - in the present case $\left|K^{0}\right\rangle$ whereas $|\mathbf{2}\rangle$ corresponds to the antiparticle state for particle "1": $\left|\bar{K}^{0}\right\rangle,\langle\mathbf{j} \mid \mathbf{k}\rangle=\delta_{j k}, j, k=1,2$. It is usually assumed that the real parts of the diagonal matrix elements of $H_{\|}$namely $\Re(\cdot)$,

$$
\mathfrak{R}\left(h_{j j}\right) \equiv M_{j j} \quad(j=1,2),
$$

where

$$
h_{j k}=\left\langle\mathbf{j}\left|H_{\|}\right| \mathbf{k}\right\rangle \quad(j, k=1,2)
$$

correspond to the masses of the particle " 1 " and its antiparticle "2" [3-7]. $\Im(\cdot)$ is the imaginary part of $h_{j j}$

$$
\mathfrak{I}\left(h_{i j}\right) \equiv \Gamma_{j j} \quad(j=1,2)
$$

and $\Gamma_{j j}$ are interpreted as the decay widths of the particles. According to the standard result of the LOY approach, in a CPT invariant system, i.e. when

$$
\Theta H \Theta^{-1}=H,
$$

(where $\Theta=\mathrm{CPT}, H=H^{+}$is the Hamiltonian of the total system under consideration) we have

$$
h_{11}^{\mathrm{LOY}}=h_{22}^{\mathrm{LOY}} .
$$

The universal properties of the unstable particle-antiparticle subsystem described by the $H$ fulfilling the condition (10), may be investigated by using the matrix elements of the exact $U_{\|}$, instead of the approximate one used in the LOY theory. The exact $U_{\|}$can be written as follows

$$
U_{\|}(t)=P U(t) P
$$

where

$$
P \equiv|\mathbf{1}\rangle\langle\mathbf{1}|+| \mathbf{2}\rangle\langle\mathbf{2}|,
$$

and $U(t)$ is the exact evolution operator acting in the whole state space. This operator is the solution of the Schrödinger equation

$$
i \frac{\partial}{\partial t} U(t)|\phi\rangle=H U(t)|\phi\rangle, \quad U(0)=I .
$$

$I$ is the unit operator in the $\mathcal{H}$ space and $|\phi\rangle \equiv\left|\phi ; t_{0}=0\right\rangle \in \mathcal{H}$ is the initial state of the system.

In the remaining part of the paper we will be using the following matrix representation of the evolution operator

$$
U_{\|}(t) \equiv\left(\begin{array}{cc}
\mathbf{A}(\mathbf{t}) & \mathbf{0} \\
\mathbf{0} & \mathbf{0}
\end{array}\right),
$$

where $\mathbf{0}$ denotes the zero submatrices of the suitable dimension, and the $\mathbf{A}(\mathbf{t})$ is a $(2 \times 2)$ matrix acting in $\mathcal{H}_{4}$

$$
A(t)=\left(\begin{array}{ll}
A_{11}(t) & A_{12}(t) \\
A_{21}(t) & A_{22}(t)
\end{array}\right),
$$

where

$$
A_{j k}(t)=\left\langle\mathbf{j}\left|U_{\|}(t)\right| \mathbf{k}\right\rangle \equiv\langle\mathbf{j}|U(t)| \mathbf{k}\rangle \quad(j, k=1,2) .
$$

Assuming that the property (10) holds and using the following definitions

$$
\Theta|\mathbf{1}\rangle \equiv e^{-i \theta}|\mathbf{2}\rangle, \quad \Theta|\mathbf{2}\rangle \equiv e^{-i \theta}|\mathbf{1}\rangle,
$$

it can be shown that

$$
A_{11}(t)=A_{22}(t)
$$


A very important relation between the amplitudes $A_{12}(t)$ and $A_{21}(t)$ follows from the famous Khalfin Theorem [8-10]

$$
r(t) \equiv \frac{A_{12}(t)}{A_{21}(t)}=\mathrm{const} \equiv r \Rightarrow|r|=1 .
$$

General conclusions concerning the properties of the matrix elements of $H_{\|}$can be drawn by analyzing the following identity $[2,5]$

$$
H_{\|}(t) \equiv i \frac{\partial \mathbf{A}(t)}{\partial t}[\mathbf{A}(t)]^{-1}
$$

Using Eq. (21) we can easily find the general formulae for the diagonal matrix elements $h_{j j}$, of $H_{\|}(t)$ and next assuming (10) and using relation (19) which follows from our earlier assumptions, we get

$$
\begin{gathered}
h_{11}(t)-h_{22}(t)= \\
=\frac{i}{\operatorname{det} \mathbf{A}(t)}\left(\frac{\partial A_{21}(t)}{\partial t} A_{12}(t)-\frac{\partial A_{12}(t)}{\partial t} A_{21}(t)\right) .
\end{gathered}
$$

In [2] it was shown, by using relation (22), that this result means that in the considered case (with CPT conserved) for $t>0$ we get the following theorem

$$
h_{11}(t)-h_{22}(t)=0 \Leftrightarrow \frac{A_{12}(t)}{A_{21}(t)}=\text { const } \quad(t>0) .
$$

Thus, for $t>0$ the problem under study is reduced to the Khalfin Theorem (see relation (20)) [2].

Having noticed this, let us now turn our attention to the conclusions following from Khalfin's Theorem. $C P$ noninvariance requires that $|r| \neq 1[3-6,8,10,12,13]$. This means that in this case the following condition must be fulfilled: $r=r(t) \neq$ const. Consequently, if in the considered system property (10) holds, but at the same time

$$
[\mathcal{C P}, H] \neq 0
$$

and the unstable states "1" $i$ "2" are connected by (18), then in this system for $t>0$ [2]

$$
h_{11}(t)-h_{22}(t) \neq 0 \text {. }
$$

So, in the exact quantum theory the difference $\left(h_{11}(t)-h_{22}(t)\right)$ cannot be equal to zero with CPT conserved and CP violated.

\section{A MODEL: ONE POLE APPROXIMATION}

While describing the two and three pion decay we are mostly interested in the $\left|K_{S}\right\rangle$ and $\left|K_{L}\right\rangle$ superposition of $\left|K^{0}\right\rangle$ and $\left|\bar{K}^{0}\right\rangle$. These states correspond to the physical $\left|K_{S}\right\rangle$ and $\left|K_{L}\right\rangle$ neutral kaon states $[13,14]$

$$
\left|K_{S}\right\rangle=p\left|K^{0}\right\rangle+q\left|\bar{K}^{0}\right\rangle, \quad\left|K_{L}\right\rangle=p\left|K^{0}\right\rangle-q\left|\bar{K}^{0}\right\rangle .
$$

Using the spectral formalism we can write an unstable state $|\lambda(t)\rangle$ as

$$
|\lambda(t)\rangle=\sum_{q}|q(t)\rangle \omega_{\lambda}(q)
$$

where $|q(t)\rangle=e^{-i t H}|q\rangle$, vectors $|q\rangle$ form a complete set of eigenvectors of the hermitian, quantum-mechanical Hamiltonian $H$ and $\omega_{\lambda}(q)=\langle q \mid \lambda\rangle$. If the continuous eigenvalue is denoted by $m$, we can define the survival amplitude $A(t)$ (or the transition amplitude in the case $\left.K^{0} \leftrightarrow \bar{K}^{0}\right)$ of ) in the following way:

$$
A(t)=\int_{\operatorname{Spec}(H)} d m e^{-i m t} \rho(m),
$$

where the integral extends over the whole spectrum of the Hamiltonian and density $\rho(m)$ is defined as follows

$$
\rho(m)=\left|\omega_{\lambda}(m)\right|^{2},
$$

where $\omega_{\lambda}(m)=\langle m \mid \lambda\rangle$.

In accordance with formula (27) the unstable states $K_{S}$ and $K_{L}$ may now be written as a superposition of the eigenkets

$$
\begin{gathered}
\left|K_{S}\right\rangle=\int_{0}^{\infty} d m \sum_{a} \omega_{S, \alpha}(m)\left|\phi_{\alpha}(m)\right\rangle \\
\left|K_{L}\right\rangle \int_{0}^{\infty} d m \sum_{\beta} \omega_{L, \beta}(m)\left|\phi_{\beta}(m)\right\rangle .
\end{gathered}
$$

The Breit-Wigner ansatz [15]

$$
\rho_{B W}(m)=\frac{\Gamma}{2 \pi} \frac{1}{\left(m-m_{0}\right)^{2}+\frac{\Gamma^{2}}{4}} \equiv|\omega(m)|^{2}
$$

leads to the well known exponential decay law which follows from the survival amplitude

$$
A_{B W}(t)=\int_{-\infty}^{\infty} d m e^{-i m t} \rho_{B W}(m)=e^{-i m_{0} t} e^{-\frac{1}{2} \Gamma|t|} .
$$

(Note that the existence of the ground state induces non-exponential corrections to the decay law and to the survival amplitude (33) - see [13]). It is therefore reasonable to assume a suitable form for $\omega_{S, \beta}$ and $\omega_{L, \beta}$. More specifically, we write [13]

$$
\begin{aligned}
& \omega_{S, \beta}(m)=\sqrt{\frac{\Gamma S}{2 \pi}} \frac{A_{S, \beta}\left(K_{S} \rightarrow \beta\right)}{m-m_{S}+i \frac{\Gamma_{S}}{2}}, \\
& \omega_{L, \beta}(m)=\sqrt{\frac{\Gamma_{L}}{2 \pi}} \frac{A_{L, \beta}\left(K_{L} \rightarrow \beta\right)}{m-m_{L}+i \frac{\Gamma_{L}}{2}},
\end{aligned}
$$


where $A_{S, \beta}$ and $A_{L, \beta}$ are decay (transition) amplitudes, and thus

$$
\rho_{x, \beta}(m)=\frac{\Gamma_{x}}{2 \pi} \frac{\left(A_{x, \beta}\left(K_{x} \rightarrow \beta\right)\right)^{2}}{\left(m-m_{x}\right)^{2}+\frac{\left(\Gamma_{x}\right)^{2}}{4}}
$$

where $x=L, S$.

In the one-pole approximation (34) $A_{K^{0} K^{0}}(t)$ can be conveniently written as

$$
\begin{aligned}
& A_{K^{0} K^{0}}(t)=A_{\bar{K}^{0} \bar{K}^{0}}(t)= \\
& =-\frac{1}{2 \pi}\left\{e^{-i m_{s} t}\left(-\int_{0}^{-\frac{m_{s}}{\gamma_{s}}} d y \frac{e^{-i \gamma_{s} t y}}{y^{2}+1}+\int_{0}^{\infty} d y \frac{e^{-i \gamma_{s} t y}}{y^{2}+1}\right)+\right. \\
& +e^{-i m_{L} t}\left(-\int_{0}^{-\frac{m_{L}}{\gamma_{L}}} d y \frac{e^{-i \gamma_{L} t y}}{y^{2}+1}+\int_{0}^{\infty} d y \frac{e^{-i \gamma_{L} t y}}{y^{2}+1}\right) .
\end{aligned}
$$

Collecting only exponential terms in (36) one obtains an expression analogous to the WW approximation [13]

$$
\begin{gathered}
A_{K^{0} K^{0}}(t)=A_{\bar{K}^{0} \bar{K}^{0}}(t)= \\
=\frac{1}{2}\left(e^{-i m_{S} t} e^{-\gamma_{S} t}+e^{-i m_{L} t} e^{-\gamma_{L} t}\right)+N_{K^{0} K^{0}}(t) .
\end{gathered}
$$

Here $N_{K^{0} K^{0}}(t)$ denotes all non-oscillatory terms contained in an exponential integral function $E_{i}$ appearing in the integral (36).

\section{DIAGONAL MATRIX ELEMENTS OF THE EFFECTIVE HAMILTONIAN}

Using the decomposition of type (37) and the one-pole ansatz (34), we find the difference (25), which is now formulated for the $K^{0}-\bar{K}^{0}$ system. Here it has the following form:

$$
h_{11}(t)-h_{22}(t)=\frac{X(t)}{Y(t)}
$$

where

$$
X(t)=i\left(\frac{\partial A_{\bar{K}^{0} K^{0}}(t)}{\partial t} A_{K^{0} \bar{K}^{0}}(t)-\frac{\partial A_{K^{0} \bar{K}^{0}}(t)}{\partial t} A_{\bar{K}^{0} K^{0}}(t)\right)
$$

and

$$
Y(t)=A_{K^{0} K^{0}}(t) A_{\bar{K}^{0} \bar{K}^{0}}(t)-A_{K^{0} \bar{K}^{0}}(t) A_{\bar{K}^{0} K^{0}}(t) .
$$

Using the above mentioned spectral formulae in the onepole approximation (34) we get $A_{K^{0} \bar{K}^{0}}(t)$ and $A_{\bar{K}^{0} K^{0}}(t)$

$$
\begin{gathered}
A_{K^{0} \bar{K}^{0}}(t)=\frac{1+\pi}{8 \pi p^{*} q}\left\{e^{-i m_{S} t} e^{-\gamma_{S} t}[1+\right. \\
\left.+\frac{\sqrt{\gamma_{S} \gamma_{L}}}{\gamma_{S}}\left(-2 i \gamma_{S} C_{I}-D_{I}^{\prime}-F_{I}^{\prime}\right]\right]+e^{-i m_{L} t e^{-\gamma_{L} t}[-1+} \\
\left.\left.+\frac{\sqrt{\gamma_{S} \gamma_{L}}}{\gamma_{L}}\left(2 i \gamma_{L} C_{I}-D_{I}^{\prime}+F_{I}^{\prime}\right)\right]\right\}+N_{K^{0} \bar{K}^{0}}(t),
\end{gathered}
$$

where the expression $N_{K^{0} \bar{K}^{0}}(t)$ is non-oscillatory terms containing the exponential integral function $E_{i}$ and it has following form

$$
\begin{aligned}
& N_{K^{0} \bar{K}^{0}}(t)=\frac{1}{8 \pi i p^{*} q}\left\{e^{-i m_{S} t} e^{-\gamma_{S} t} E_{i}\left(\gamma_{S} t+i m_{S} t\right)(1+\right. \\
& \left.+\sqrt{\gamma_{S} \gamma_{L}}\left[-2 i C_{I}+\frac{1}{\gamma_{S}}\left(D_{I}^{\prime}-F_{I}^{\prime}\right)\right]\right)+ \\
& +e^{-i m_{L} t} e^{-\gamma_{L} t} E_{i}\left(\gamma_{L} t+i m_{L} t\right)(-1+ \\
& \left.+\sqrt{\gamma_{S} \gamma_{L}}\left[2 i C_{I}+\frac{1}{\gamma_{L}}\left(-D_{i}^{\prime}+F_{i}^{\prime}\right)\right]\right)+ \\
& +e^{-i m_{S} t} e^{\gamma_{S} t} E_{i}\left(-\gamma_{S} t+i m_{S} t\right)(1+ \\
& \left.+\sqrt{\gamma_{S} \gamma_{L}}\left[2 i C_{I}+\frac{1}{\gamma_{S}}\left(-D_{i}^{\prime}+F_{i}^{\prime}\right)\right]\right)+ \\
& +e^{-i m_{L} t} e^{\gamma_{L} t} E_{i}\left(-\gamma_{L} t+i m_{L} t\right)(-1+ \\
& \left.\left.+\sqrt{\gamma_{S} \gamma_{L}}\left[2 i C_{I}+\frac{1}{\gamma_{L}}\left(D_{I}^{\prime}-F_{I}^{\prime}\right)\right]\right)\right\}
\end{aligned}
$$

and

$$
\begin{gathered}
A_{\bar{K}^{0} K^{0}}(t)=\frac{1+\pi}{8 \pi p q^{*}}\left\{e^{-i m_{S} t} e^{-\gamma_{S} t}[1+\right. \\
\left.+\frac{\sqrt{\gamma_{S} \gamma_{L}}}{\gamma_{S}}\left(2 i \gamma_{S} C_{I}-D_{I}^{\prime}+F_{I}^{\prime}\right)\right]+ \\
+e^{-i m_{L} t} e^{-\gamma_{L} t}[-1+
\end{gathered}
$$




$$
\begin{aligned}
&+\frac{\sqrt{\gamma_{S} \gamma_{L}}}{\gamma_{L}}(\left.\left.\left.-2 i \gamma_{L} C_{I}+D_{I}^{\prime}-F_{I}^{\prime}\right)\right]\right\}+ \\
&+N_{\bar{K}^{0} K^{0}}(t),
\end{aligned}
$$

where $N_{\bar{K}^{0} K^{0}}(t)$ denotes all non-oscillatory terms of the form similar to (42) and $C_{I}, D_{I}^{\prime}, F_{I}^{\prime}$ are defined in [13].

Using the expression for the derivative of $E_{i}$ we can find the derivatives which will be necessary for the following calculations $\frac{\partial A_{K^{0} \bar{K}^{0}}(t)}{\partial t}$ and $\frac{\partial A_{\bar{K}^{0} K^{0}}(t)}{\partial t}$ :

$$
\begin{gathered}
\frac{\partial A_{K^{0} \bar{K}^{0}}(t)}{\partial t}=\frac{1+\pi}{8 \pi p^{*} q}\left\{e ^ { - i m _ { S } t } e ^ { - \gamma _ { S } t } \left[-i m_{S}-\gamma_{S}+\right.\right. \\
\left.+\sqrt{\gamma_{S} \gamma_{L}}\left(2 i \gamma_{S} C_{I}-D_{I}^{\prime}+F_{I}^{\prime}\right)\right]+ \\
+e^{-i m_{L} t} e^{-\gamma_{L} t}\left[i m_{L}-\gamma_{L}+\right. \\
\left.\left.+\sqrt{\gamma_{S} \gamma_{L}}\left(-2 i \gamma_{L} C_{I}+D_{I}^{\prime}-F_{I}^{\prime}\right)\right]\right\}+ \\
+\Delta N_{K^{0} \bar{K}^{0}}(t)
\end{gathered}
$$

where $\Delta N_{K^{0} \bar{K}^{0}}(t)$ is defined as follows

$$
\begin{aligned}
\Delta N_{K^{0} \bar{K}^{0}}(t) & =\frac{1}{8 \pi i p^{*} q}\left\{e ^ { - i m _ { S } t } e ^ { - \gamma _ { S } t } E _ { i } ( \gamma _ { S } t + i m _ { S } t ) \left(-i m_{S}+\right.\right. \\
& \left.-\gamma_{S}+\sqrt{\gamma_{S} \gamma_{L}}\left(2 i \gamma_{S} C_{I}-D_{I}^{\prime}+F_{I}^{\prime}\right)\right)+ \\
+ & e^{-i m_{L} t} e^{-\gamma_{L} t} E_{i}\left(\gamma_{L} t+i m_{L} t\right)\left(i m_{L}-\gamma_{L}+\right. \\
+ & \left.\sqrt{\gamma_{S} \gamma_{L}}\left(-2 i \gamma_{L} C_{I}+D_{I}^{\prime}-F_{I}^{\prime}\right)\right)+ \\
+ & e^{-i m_{S} t} e^{\gamma_{S} t} E_{i}\left(-\gamma_{S} t+i m_{S} t\right)\left(i m_{S}-\gamma_{S}+\right. \\
& \left.+\sqrt{\gamma_{S} \gamma_{L}}\left(-2 i \gamma_{S} C_{I}-D_{I}^{\prime}+F_{I}^{\prime}\right)\right)+ \\
+ & e^{-i m_{L} t} e^{\gamma_{L} t} E_{i}\left(-\gamma_{L} t+i m_{L} t\right)\left(-i m_{L}-\gamma_{L}+\right. \\
& \left.\left.+\sqrt{\gamma_{S} \gamma_{L}}\left(2 i \gamma_{L} C_{I}+D_{I}^{\prime}-F_{I}^{\prime}\right)\right)\right\} .
\end{aligned}
$$

$$
\begin{gathered}
\frac{\partial A_{\bar{K}^{0} K^{0}}(t)}{\partial t}=\frac{1+\pi}{8 \pi p^{*} q}\left\{e ^ { - i m _ { S } t } e ^ { - \gamma _ { S } t } \left[-i m_{S}-\gamma_{S}+\right.\right. \\
\left.+\sqrt{\gamma_{S} \gamma_{L}}\left(-2 i \gamma_{S} C_{I}+D_{I}^{\prime}-F_{I}^{\prime}\right)\right]+ \\
+e^{-i m_{L} t} e^{-\gamma_{L} t}\left[i m_{L}-\gamma_{L}+\right. \\
\left.\left.+\sqrt{\gamma_{S} \gamma_{L}}\left(2 i \gamma_{L} C_{I}-D_{i}^{\prime}+F_{I}^{\prime}\right)\right]\right\}+ \\
+\Delta N_{\bar{K}^{0} K^{0}}(t),
\end{gathered}
$$

where analogous to (45) $\Delta N_{\bar{K}^{0} K^{0}}(t)$ denotes all non-oscillatory terms. The correctness of expressions (41)-(46) was checked using Mathematica.

The states $\left|K_{L}\right\rangle$ and $\left|K_{S}\right\rangle$ are superpositions of $\left|K^{0}\right\rangle$ and $\left|\bar{K}^{0}\right\rangle$. The lifetimes of particles $\left|K_{L}\right\rangle$ and $\left|K_{S}\right\rangle$ may be denoted by $\tau_{L}$ and $\tau_{S}$, respectively, $\tau_{L}=\frac{1}{\gamma_{L}}=5.183 \cdot 10^{-8} \mathrm{~s}$ being much longer than $\tau_{S}=\frac{1}{\gamma_{s}}=0.8923 \cdot 10^{-10} \mathrm{~s}$.

Below we calculate the difference (38) for $t \sim \tau_{L}$

$$
h_{11}\left(t \sim \tau_{L}\right)-h_{22}\left(t \sim \tau_{L}\right)=\frac{X\left(t \sim \tau_{L}\right)}{Y\left(t \sim \tau_{L}\right)} .
$$

If we only consider the long living states $\left|K_{L}\right\rangle$ we may drop all the terms containing $\left.e^{-\gamma_{S} t}\right|_{t \sim \tau_{L}}$ as they are negligible in comparison with elements involving the factor $\left.e^{-\gamma_{L} t}\right|_{t \sim \tau_{L}}$. We also drop all the non-oscillatory terms $N_{K^{0} K^{0}}(t), \quad N_{\bar{K}^{0} K^{0}}(t), \quad N_{K^{0} \bar{K}^{0}}(t)$ present in $A_{K^{0} K^{0}}(t)$, $A_{\bar{K}^{0} K^{0}}^{K^{0} K^{0}}(t)$ and $A_{K^{0} \bar{K}^{0}}(t)$, that is in integrals (36), (41) and (43), because they are extremally small in the region of time $t \sim \tau_{L}[13,16,17]$. Similarly, because of the properties of the exponential integral function $E_{i}$, we can drop terms like $\Delta N_{\bar{K}^{0} K^{0}}$ and $\Delta N_{K^{0} \bar{K}^{0}}$ present in $\frac{\partial A_{\bar{K}^{0} K^{0}}}{\partial t}$ (44) and $\frac{\partial A_{K^{0} K^{0}}}{\partial t}$ (46). This conclusion follows from the asymptotic properties of the exponential integral function $E_{i}$ (see formulae (53) in Appendix A) and the fact that $\Delta N_{\bar{K}^{0} K^{0}}$, $\Delta N_{K^{0} \bar{K}^{0}}$ contain only expressions proportional to $E_{i}$. Simply, from (53) it follows that terms of type $e^{-x} E_{i}(x)$ are negligible in comparison with $e^{-x}$ for $|x| \rightarrow \infty$ and $\operatorname{Re} x>0$.

We may now calculate the products $A_{K^{0} K^{0}}(t) A_{\bar{K}^{0} \bar{K}^{0}}(t)$, $A_{K^{0} \bar{K}^{0}}(t) A_{\bar{K}^{0} K^{0}}(t), \frac{\partial A_{\bar{K}^{0} K^{0}}}{\partial t}(t) A_{K^{0} \bar{K}^{0}}(t), \frac{\partial A_{K^{0} \bar{K}^{0}}}{\partial t}(t) A_{\bar{K}^{0} K^{0}}(t)$, which, after using the above mentioned properties of $N_{K^{0} K^{0}}(t)$, $\Delta N_{K^{0} K^{0}}(t)$ and performing some algebraic transformations, leads to the following form of the difference (47)

$$
h_{11}\left(t \sim \tau_{L}\right)-h_{22}\left(t \sim \tau_{L}\right)=\left(\frac{2 \pi^{2} \sqrt{\gamma_{S} \gamma_{L}}}{\pi^{2}+2 \pi+1}\right) \cdot \frac{Z}{W} \neq 0,
$$




$$
\begin{gathered}
Z=4|p|^{2}|q|^{2}-\frac{\pi^{2}+2 \pi+1}{4 \pi^{2}}[1+ \\
+\gamma_{S}\left(4 \gamma_{L} C_{I}^{2}+\frac{1}{\gamma_{L}}\left(-D_{I}^{\prime 2}-F_{I}^{\prime 2}+4 D_{I}^{\prime} F_{I}^{\prime}\right)+\right. \\
\left.+4 i C_{I}\left(D_{I}^{\prime}-F_{I}^{\prime}\right)\right] \neq 0 \\
W=2\left(-C_{I} m_{L}+D_{I}^{\prime}-F_{I}^{\prime}\right)+ \\
+i\left[-4 C_{I} \gamma_{L}+\frac{m_{L}}{\gamma_{L}}\left(-D_{I}^{\prime}+F_{I}^{\prime}\right)\right] \neq 0
\end{gathered}
$$

\section{FINAL REMARKS}

Our results presented in the present paper have shown that in a CPT invariant and CP noninvariant system in the case of the exactly solvable one-pole model, the diagonal matrix elements do not have to be equal. In the general case the diagonal elements depend on time and their difference, for example at $t \sim \tau_{L}$, is different from zero. $Z$ and $W$ in (48) are different from zero, so the difference $\left.\left(h_{11}(t)-h_{22}(t)\right)\right|_{t \sim \tau_{L}} \neq 0$. From this observation a conclusion of major importance can be drawn, namely that the measurement of the mass difference $\left(m_{K^{0}}-m_{\bar{K}^{0}}\right)$ should not be used while designing CPT invariance tests. This runs counter to the general conclusions following from the Lee, Oehme and Yang theory.

A detailed analysis of $h_{j k}(t),(j, k=1,2)$ shows that the non-oscillatory elements $N_{\alpha, \beta}(t), \Delta N_{\alpha, \beta}(t) \quad$ (where $\left.\alpha, \beta=K^{0}, \bar{K}^{0}\right)$ is the source of the non-zero difference $\left(h_{11}(t)-h_{22}(t)\right)$ in the model considered. It is not difficult to verify that dropping all the terms of $N_{\alpha, \beta}(t), \Delta N_{\alpha, \beta}(t)$ type in the formula for $\left(h_{11}(t)-h_{22}(t)\right)$ gives $\left(h_{11}^{\text {osc }}(t)-h_{22}^{\text {osc }}(t)\right)=0$, where $h_{j j}^{\text {osc }}(t),(j=1,2)$, stands for $h_{j j}(t)$ without the non-oscillatory terms.

The result $\left(h_{11}(t)-h_{22}(t)\right) \neq 0$ seems to be very important as it has been obtained within the exactly solvable one-pole model based on the Breit-Wigner ansatz, i.e. the same model as used by Lee, Oehme and Yang.

The next future step is to search for properties of $\left(h_{11}(t)-h_{22}(t)\right)$ for time varying from $t=0$ to $t \gg \tau_{L}$. This problem one can solve using Mathematica or other computer programs.

\section{Acknowledgements}

The author wishes to thank Professor Krzysztof Urbanowski for many helpful discussions.

\section{Appendix A}

This appendix contains the relevant properties of the exponential integral function $E_{i}$ used in this paper.

The exponential integral function $E_{i}$ is defined in the following way $[13,16]$

$$
E_{i}( \pm x y)= \pm e^{ \pm x y} \int_{0}^{\infty} d t \frac{e^{-x t}}{y \mp t}, \quad \Re y>0, \quad x>0 .
$$

We can use the very convenient asymptotic properties of $E_{i}$ given in [17]

$$
\begin{aligned}
& E_{i}(0)=-\infty, \\
& E_{i}(\infty)=\infty, \\
& E_{i}(-\infty)=0, \\
& E_{i}(i \infty)=i \pi, \\
& E_{i}(-i \infty)=-i \pi .
\end{aligned}
$$

$E_{i}(x)$ has following asymptotical expressions for $|x| \rightarrow \infty$ (see formulae 5.1.51 in [17])

$$
E_{i}(x) \sim \frac{e^{-x}}{x}\left\{1-\frac{1}{x}+\frac{2 !}{x^{2}}-\ldots\right\}, \quad\left(|\arg x|<\frac{3}{2} \pi\right) .
$$

These properties of $E_{i}$ have been used to obtain the final result (48)-(50).

In our calculations we have also used the formula for the derivative of $E_{i}$. Its final, general form is given below

$$
\frac{d E_{i}( \pm x y)}{d x}=\frac{1}{x} e^{ \pm x y}
$$

\section{References}

[1] H. Dehmelt, R. Mittleman, R. S. Van Dyck, Jr., P. Schwinberg, Phys. Rev. Lett. 83, 4694 (1999).

[2] K. Urbanowski, Phys. Lett., B 540, 89 (2002).

[3] T. D. Lee, R. Oehme, C. N. Yang, Phys. Rev., 106, 340 (1957).

[4] T. D. Lee, C. S. Wu, Annu. Rev. Nucl. Sci., 16, 471 (1966); M. K. Gaillard, M. Nicolic (Eds.), Weak Interaction, INPN et de Physique des Particules, Paris, 1977, Ch. 5, Appendix A; S. M. Bilenkij, in: Particles and Nucleus, Vol. 1. (1), 1970, p. 227, [in Russian].

[5] L. P. Horwitz, J. P. Marchand, Helv. Phys. Acta, 42, 801 (1969).

[6] J. W. Cronin, Acta Phys. Polon., B 15, 419 (1984); V. V. Barmin et al., Nucl. Phys., B 247, 428 (1984); L. Lavoura, Ann. Phys. (N.Y.), 207, 428 (1991); C. Buchanan et al., Phys. Rev., D 45, 4088 (1992); C. O. Dib, R. D. Peccei, Phys. Rev., D 46, 2265 (1992); M. Zrałek, Acta Phys. Polon., B 29, 3925 (1998); M. Nowakowski, Mod. Phys. Lett. A 17, 2039 (2002).

[7] K. Urbanowski, J. Piskorski, Found. Phys., 30, 839 (2000), physics/9803030.

[8] L. A. Khalfin, Preprints of the University of Texas at Austin: New Results on the CP-violation problem (Report DOE-ER40200-211, February 1990); L. A. Khalfin, A new $C P$-violation effect and new possibility for investigation of $K_{S}^{0}, K_{L}^{0}\left(K^{0}, \bar{K}^{0}\right)$ decay modes (Report DOE-ER40200-247, Frebruary 1991).

[9] P. K. Kabir, A. Pilaftsis, Phys. Rev., A 53, 66 (1996). 
[10] L. A. Khalfin, Found. Phys., 27, 1549 (1997), and references one can find therein.

[11] O. Nachtmann, Elementary Particle Physics, Springer Verlag, Berlin 1990.

[12] K. Hagiwara et al., Review of Particle Physics, Physical Review D 66, Part 1, No 1--I, 010001 (2002).

[13] M. Nowakowski, Time Evolution of $K^{0}-\bar{K}^{0}$ System in Spectral Formulation, SIS-Pubblicazioni, LNF-96/004(P); M. Nowakowski, Int. J. Mod. Phys., A 14, 589 (1999) .

[14] P. K. Kabir, The CP Puzzle, Academic Press, London 1968.
[15] A. Bohm, Quantum Mechanics: Foundations and Applications, Springer Verlag, Berlin 1986.

[16] I. S. Gradshteyn, I. M. Ryzhik, Tables of Integrals, Series and Products, 4th edition, Academic Press, London 1965.

[17] M. Abramowitz and I. A. Stegun, Handbook of Mathematical Functions with Formulas, Graphs, and Mathematical Tables, National Bureau of Standards Applied Mathematics Series 55, Issued June 1964 Tenth Printing, December 1972, with corrections.

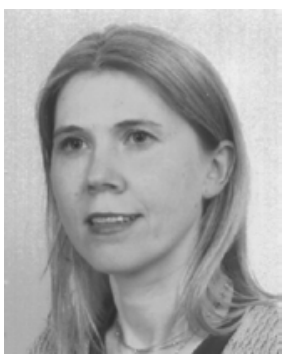

JUSTYNA JANKIEWICZ studied physics and received the master's degree from the Pedagogical University, Zielona Góra, Poland. Since 1999, she has been with the Theory of Fundamental Interactions Department, Institute of Physics, University of Zielona Góra, where she is currently an Assistant. Her research interests focus on the properties of time evolution of unstable neutral particles (neutral K mesons). 